This item was submitted to Loughborough's Research Repository by the author.

Items in Figshare are protected by copyright, with all rights reserved, unless otherwise indicated.

\title{
Impedance study on oxygen diffusion through fuel cell cathode catalyst layer at high current
}

\section{PLEASE CITE THE PUBLISHED VERSION}

http://dx.doi.org/10.1149/1.3502569

\section{PUBLISHER}

(C) Electrochemical Society

\section{VERSION}

AM (Accepted Manuscript)

\section{PUBLISHER STATEMENT}

This work is made available according to the conditions of the Creative Commons Attribution-NonCommercialNoDerivatives 4.0 International (CC BY-NC-ND 4.0) licence. Full details of this licence are available at: https://creativecommons.org/licenses/by-nc-nd/4.0/

\section{LICENCE}

CC BY-NC-ND 4.0

\section{REPOSITORY RECORD}

Cruz-Manzo, Samuel, Pratap Rama, and Rui Chen. 2015. "Impedance Study on Oxygen Diffusion Through Fuel Cell Cathode Catalyst Layer at High Current”. figshare. https://hdl.handle.net/2134/18547. 


\title{
Impedance Study on Oxygen Diffusion through Fuel Cell Cathode Catalyst Layer at High
}

\section{Current}

\author{
Samuel Cruz-Manzo, Pratap Rama, Rui Chen* \\ Loughborough University, United Kingdom
}

\begin{abstract}
A mathematical model to simulate the electrochemical impedance spectrum in the frequency domain and the current distribution in the time domain of polymer electrolyte fuel cell (PEFC) cathode catalyst layer (CCL) operated at high currents has been developed. In the model, the Fick's second law in the frequency domain is solved to define oxygen distribution through CCL. The rate of oxygen transportation and proton conductivity are related to the current distribution equation reported in the authors' previous study for low current operations. The model are compared against the frequency response of an experimental impedance spectrum, is then converted into the time domain using the inverse Laplace transform method. The results show the non-steady oxygen diffusion in the CCL which allows equilibrium to be established between the bulk concentration supplied at the GDL boundary and the surface concentration of the oxygen within the CCL. The developed model can be applied to unveil the effect of kinetic, ohmic, and mass transport mechanisms on current distribution through the thickness of the cathode catalyst layer (CCL) from the measured impedance results.
\end{abstract}

*Correspondence author: r.chen@1boro.ac.uk 
Polymer Electrolyte Fuel Cells (PEFC) generate electrical and thermal energy by combining hydrogen and oxygen. The core of the PEFC is the Membrane Electrode Assembly (MEA), which consists of a Proton Exchange Membrane (PEM), anodic and Cathodic Catalyst Layers (CCL), and anodic and cathodic Gas Diffusion Layers (GDL). A catalyst layer typically contains carbon-supported catalytic particles (electrode) and a matrix of polymer electrolyte (electrolyte). Structurally, it contains a tortuous pore network for the supply and removal of reactant gases and product water, respectively, and proton and electron conducting networks for ionic transport [1]. The performance of a PEFC depends on the concentration of the reactants supplied to the catalyst layer. A concentration gradient is therefore established which affects the electrochemical reaction. The study of the mass transport in the CCL is complex, yet has been treated in the literature with certain simplifications and approximations. Berger et al. $[2,3,4,5,6]$ considers that diffusion occurs perpendicularly to the surface of the electrochemical reaction. Under this condition is possible to assume that the drop of the reactant concentration is linear. There are two different methods of describing species diffusion in electrochemical systems when a thin film of finite diffusion is involved, such as in the CCL. Finite diffusion in the CCL in the first, which describes the slow diffusion of the oxygen through a coating or a passive film. Semi-infinite diffusion is another form and can occur in batteries or super capacitors [7]. This method relies on a fixed amount of electro-active material; once it is consumed, it cannot be replaced.

Electrochemical Impedance Spectroscopy (EIS) is a technique that can be applied in-situ to separate the effects of physical processes in the PEFC that occur at different rates and to characterise mass transport effects. The frequency response that results from the EIS technique can be represented by an equivalent electrical circuit that is composed of resistors and capacitors. Each component represents a physical phenomenon occurring inside the PEFC. The literature demonstrates that the Warburg element in an equivalent electrical circuit of the PEFC can account for diffusive oxygen transport in the CCL, while the use of the Warburg element to model semi-infinite and finite diffusion in electrochemical systems has also been demonstrated. Dzmitry et al. [8] used the EIS technique and applied the Warburg element for finite diffusion in the equivalent circuit of a PEFC to study the influence of mass transport in the Micro-Porous Layer (MPL) of the cathode. The results showed that mass transport resistance increases with high current density for cells with and without the MPL. However, cells with a MPL demonstrated a lower mass-transport resistance compared to cells without a MPL. Skale et al. [9] proposed an alternative model for fitting electrochemical impedance spectra of protective coatings. EIS measurements were applied to characterize the coating degradation which is important to reduce the corrosion rate of metals. In this work the Warburg element for a semi-infinite diffusion in combination with EIS data were used to analyze the ion diffusion through micropores in the coating. That work concluded that the use of the Warburg element presents a better fit for these types of measurements than by models employing the constant phase element (CPE). The use of the semi-infinite Warburg impedance has also been used by Onda [7] to approximate the response of nickel/metal-hydride and lithium-ion batteries. EIS measurements with the pulse current method and the equivalent circuit could 
provide a good estimate of the overpotential resistance that describes the thermal behaviour of batteries. Ciunerau M. [10] applied EIS measurements with an equivalent electrical circuit which contains the semi-infinite Warburg impedance to measure the proton conductivity of hydrated membranes of the PEFC. The EIS spectra showed a straight line with a $45^{\circ}$ slope for the pure diffusion case. At high temperatures the diffusion component of the spectra decreased, which can be attributed to dehydration. Fouquet monitored flooding and drying of a PEFC using a Randless electrical circuit coupled with a Warburg finite-length diffusion element in combination with ac impedance measurements [11]. These results showed that the electrical model allowed parameters exhibiting high sensitivity to either flooding or drying out of the MEA to be identified.

The objective of the current research is to develop a mathematical model to simulate the high current impedance spectrum in the frequency domain and the corresponding spatio-temporal current and oxygen distribution through the CCL using diffusion theory and the fundamental theory of the electrochemistry. It is possible as such to establish a wider scope to relate the AC electrochemical impedance technique to the fundamental theory of PEFCs.

\section{Transport Mechanisms in Frequency Domain}

The catalyst layer is formed by a double layer structure between the electrode and electrolyte interface. This double layer can behave like a capacitor that is in parallel with the electrode reactions. Therefore the current across the thickness of the cathode catalyst layer can take part in the charge transfer due to the oxygen reduction reaction (ORR) or contribute to the charge in the capacitive effect. This phenomenon was discussed and developed in a previous publication [12]. The electrochemical reaction in the PEFC results in an inhomogeneous distribution of charge in the catalyst layer. To correct for this inhomogeneity, a constant phase element $(\mathrm{CPE}) Y(i \omega)^{P}$, where $Y$ represents a parameter related to CPE, superscript $P$ represents a parameter to correct the inhomogeneity in the distribution of charge, $\omega$ is the angular frequency, and $i$ is the imaginary component in impedance, which is defined in the frequency domain has to be used to replace the capacitor in the current distribution equation, as such:

$\frac{d \bar{j}}{d x}=\frac{c_{O}^{\prime}}{c_{O}^{*}}\left(\frac{1}{R_{C}}\right) \bar{\eta}+Y(i \omega)^{P} \bar{\eta}$

where $\bar{j}$ is the current across the thickness of the CCL in the frequency domain, $\bar{\eta}$ is the potential difference between the electrode and electrolyte interface in the frequency domain and is defined as $\bar{\eta}=\eta_{\text {electrolyte }}-\eta_{\text {electrode }}, c_{O}^{\prime}$ is the oxygen concentration at the CCL-PEM interface, and $c_{o}^{*}$ is the bulk concentration at the GDL-CCL interface in the frequency domain, $R_{c}$ represents the charge transfer resistance presented in the ORR and is defined as $R_{C}=b / j_{0} \exp \left(\eta_{S} / b\right)$, where $b$ is the Tafel slope, $\eta_{S}$ represents a value of voltage in activation overpotential, and $j_{0}$ is the exchange current. In this work, 
the change in oxygen concentration is considered at medium and high currents, thereby addressing the assumption of $c_{O}^{\prime} / c_{O}^{*}=1$ for low current distribution. This is done by considering Fick's Second Law.

Eq.1 represents the current passing from electrode-electrolyte in the frequency domain. The first term represents the rate of charge transfer due to the distribution of oxygen and the kinetics of the ORR in the CCL. The second term represents the rate of charge transfer due to the capacitive effect in the frequency domain.

\subsection{Current Distribution in Frequency Domain}

The diffusion equation expressed by Fick's second law is considered in order to model the effect of diffusion on the distribution of oxygen concentration through the CCL with respect to time;

$\frac{\partial c_{o}(x, t)}{\partial t}=D \frac{\partial^{2} c_{o}(x, t)}{\partial x^{2}}$

The solution of Eq.2 in the Laplace domain requires an initial condition $c_{o}(x, 0)=c_{o}^{*}$ and two boundary conditions in $x$. This solution in the Laplace domain through the method of undetermined coefficients for a nonhomogeneous linear differential equation takes the form of:

$c_{O}(x, s)-c_{O}^{*}=A \exp \left(\lambda_{1} x\right)+B \exp \left(\lambda_{2} x\right)$

The characteristic equation represented in the homogeneous part of Eq.3 has two distinct roots, $\lambda_{1,2}= \pm \sqrt{s / D}$, where $D$ is the (effective) diffusion coefficient of oxygen, $s=i \omega$ is the frequency domain defined from Laplace domain. Evaluating boundary conditions in Eq.3 at the GDL-CCL $x=0$ and $c_{o}(0)=c_{o}^{*}$, while at the CCL-PEM interface $x=\delta$ and $c_{o}(\delta)=c_{o}^{\prime}$, gives :

$$
A=\frac{c_{O}^{*}-c_{O}^{\prime}}{\exp \left(\lambda_{2} \delta\right)-\exp \left(\lambda_{1} \delta\right)} \quad B=\frac{c_{O}^{\prime}-c_{O}^{*}}{\exp \left(\lambda_{2} \delta\right)-\exp \left(\lambda_{1} \delta\right)}
$$

Substituting $A$ and $B$ back into Eq.3 with trigonometric identities and relating the Faraday's law and the Fick's first law, which relates the diffusive flux to the concentration field assuming that the flux occurs from the region of high concentration to the region of low concentration, yields:

$\frac{c_{o}^{\prime}}{c_{o}^{*}}=1-\frac{d \bar{j}}{d x} \frac{\tanh (\delta \sqrt{i \omega / D})}{z F \sqrt{D} \sqrt{i \omega c_{o}^{*}}}$

where, $z$ is the number of electrons consumed during the ORR, $F$ is the Faraday constant. 
Eq.4 represents the rate of oxygen distribution in the CCL. The current $\bar{j}$ corresponds to the current that takes part in the charge transfer reaction for ORR, not to the current corresponding to the charge of the capacitive effect between electrolyte-electrode interface. When the CCL operates at low current, the second term of Eq.4 becomes negligible and the rate of oxygen distribution is aproximately equal to one. For a high current, it addresses the effect on the concentration distribution through the CCL.

With the transport of oxygen through the CCL defined via Eq.4, it is possible to define the current generated along the CCL. The use of a small amplitude sinusoidal perturbation through the EIS technique allows the use of a linear model to represent the impedance of the CCL. Substituting Eq.4 into the first term of Eq. 1 and considering $\bar{\eta}=R T / z F$ which represents a linearized relation of the overpotential, and the ideal gas constant $R$ and the operational temperature $T$ of the PEFC, yields:

$\frac{d \bar{j}}{d x}=\frac{\bar{\eta}}{R_{C}+Z_{W}}+Y(i \omega)^{P} \bar{\eta}$

where $Z_{W}$ is defined as the Warburg impedance and describes diffusion across a finite dimension in the frequency domain [11],

$Z_{W}=R_{W} \frac{\tanh \left(i \omega T_{W}\right)^{0.5}}{\left(i \omega T_{W}\right)^{0.5}}$

with

$R_{W}=\frac{R T \delta}{z^{2} F^{2} c_{o}^{*} D}$

defined as resistance for the diffusion process and

$T_{W}=\frac{\delta^{2}}{D}$

defined as the time constant to diffuse oxygen through the CCL .

The literature identifies that proton resistance in the electrolytic phase (i.e., polymer electrolyte) is higher than electron resistance presented in the electrode network of the CCL (i.e., typically carbonsupported catalyst) [13]. Furthermore proton conduction in the CCL depends upon the electrolytic dispersion and the state of hydration within the CCL. In addition, by neglecting electron conduction in the CCL as a first approximation the mathematic analysis can be simplified. The potential in the electrolyte network can then be expressed by Ohms law $d \eta_{\text {electrolyte }} / d x=\bar{j} R_{P}$, where $R_{P}$ is the resistance 
to the flow of protons in the electrolytic phase of the CCL. Substituting the Electrolyte potential into Eq.5 yields:

$$
\frac{d^{2} \bar{j}}{d x^{2}}=R_{p}\left[\frac{\bar{j}}{R_{C}+Z_{W}}+Y(i \omega)^{P} \bar{j}\right]
$$

Eq.9 is a $2^{\text {nd }}$ order homogeneous equation and represents the current distribution through the thickness of the CCL in the frequency domain taking into account spatio-temporal oxygen distribution, electrode kinetics, charge capacitance and proton resistance in the CCL. Its solution can be obtained by applying the method of the $n^{\text {th }}$-order homogeneous equation with constant coefficients:

$\bar{j}=A \exp \left(\gamma_{1} x\right)+B \exp \left(\gamma_{2} x\right)$

where $\gamma_{1,2}= \pm \sqrt{R_{p}\left[\frac{1}{R_{C}+Z_{W}}+Y(i \omega)^{P}\right]}$ represents the distinct roots of the characteristic equation represented.

Evaluating boundary conditions in Eq.10, at the GDL-CCL $x=0$ and $\bar{j}=0$, while at the CCL-PEM interface $x=L$ and $\bar{j}=j_{m}$, where $j_{m}$ represents the maximum current as a function of the proton conduction via the PEM, gives the current distribution in the frequency domain,

$$
\bar{j}=j_{m} \frac{\sinh (\gamma x)}{\sinh (\gamma L)}
$$

\subsection{Potential Distribution in Frequency Domain}

The potential in the frequency domain can be obtained by arranging Eq.5 as:

$$
\bar{\eta}=\frac{R_{C}+Z_{W}}{1+Y(i \omega)^{P}\left(R_{C}+Z_{W}\right)} \frac{d \bar{j}}{d x}
$$

Differentiating Eq. 11 and substituting into Eq.12 gives the potential in the frequency domain:

$$
\bar{\eta}=\frac{\left(R_{C}+Z_{W}\right) j_{m} \gamma \cosh (\gamma x)}{\left[1+Y(i \omega)^{P}\left(R_{C}+Z_{W}\right)\right] \sinh (\gamma L)}
$$

\section{Impedance Model of CCL}


The impedance of the CCL is defined as the ratio between the potential, Eq.13, and the current, Eq.11 at frequency domain.

$$
Z=\frac{\bar{\eta}}{\bar{j}}=\frac{\left(R_{C}+Z_{W}\right) \gamma \operatorname{coth}(\gamma x)}{1+Y(i \omega)^{P}\left(R_{C}+Z_{W}\right)}
$$

Eq.14 represents the opposition to the flow of an alternating current AC within the CCL which contains physical processes that dissipate energy and store energy. The opposition to the AC load, or impedance, can be measured over a range of frequencies, thereby revealing the frequency response of the CCL. By understanding the magnitude of the electrochemical and oxygen transport mechanisms represented in Eq.14, it is possible to generate a deeper understanding of how and where the chemical energy that is released from the redox reaction is being dissipated and retained within the CCL. The solution of Eq.14 over a range of frequencies will contain real and imaginary components, which can be presented on a Nyquist plot.

\subsection{Model Validation in Frequency Domain}

The modelling predictions of the mechanistic treatment of Eq.14 can be compared against an experimental impedance spectrum reported in the literature of a hydrogen-air PEFC $\left(\mathrm{H}_{2} /\right.$ Air $)$ operated at $700 \mathrm{~mA} / \mathrm{cm}^{2}$ and $60{ }^{\circ} \mathrm{C}$ [8]. Requisite parameters defining the kinetics of the ORR, the double charge capacitance, the bulk concentration and the (effective) diffusion coefficient of oxygen were determined directly from the published experimental data. The validation process is described here.

An experimental impedance curve measured at high current of the polarization curve was reported by Malevich et al. [8] and was considered in this study to validate the impedance model of the CCL. This author used a Randles equivalent circuit, as shown in Figure 1, to estimate the charge transfer resistance, double layer capacitance and the Warburg impedance of the CCL.

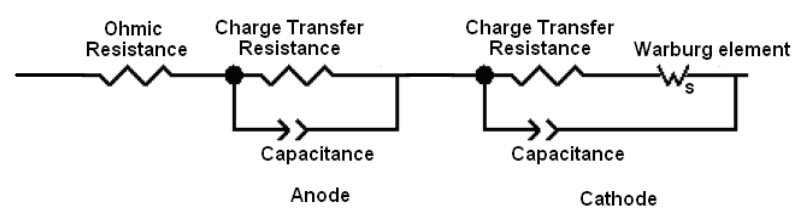

Figure 1 Randles electrical circuit of the PEFC

The parameters of the electrical circuit such as capacitance, charge transfer resistance and the Warburg element are estimated using Zview 2.9 software (Scribner Associates, Inc). As discussed in the literature [14], once an equivalent circuit model is fitted to the measured impedance spectrum of the entire cell, the electrical circuit of the anode catalyst layer and the Ohmic resistor for the PEM are all removed in order to reveal the equivalent circuit of the CCL. This can then be used to generate the AC 
impedance response of just the cathode catalyst layer in isolation, which is otherwise masked in the measured impedance spectrum of the entire cell.

In the study of Malevich et al. [8], the parameters of the CCL electrical circuit were reported and shown in table 1. These parameters were used to directly compare the model prediction from Equation 14 to the experimental spectrum of the CCL.

\begin{tabular}{|c|c|c|c|c|c|}
\hline$j \mathrm{~mA} / \mathrm{cm}^{2}$ & $R_{\text {ohm }} \Omega \mathrm{cm}^{2}$ & $R_{C} \Omega \mathrm{cm}^{2}$ & $R_{W} \Omega \mathrm{cm}^{2}$ & $T_{W} \mathrm{~s}$ & $\delta \mathrm{cm}$ \\
\hline 700 & 0.017 & 0.173 & 0.136 & 0.110 & 0.0035 \\
\hline
\end{tabular}

Table 1: Parameters of the CCL reported in the study of Malevich

Figure 2 shows the comparison between the simulated spectrum from Eq.14 and the spectrum generated by the electrical circuit of the CCL Figure 1 using the experimental data given in Table 1.

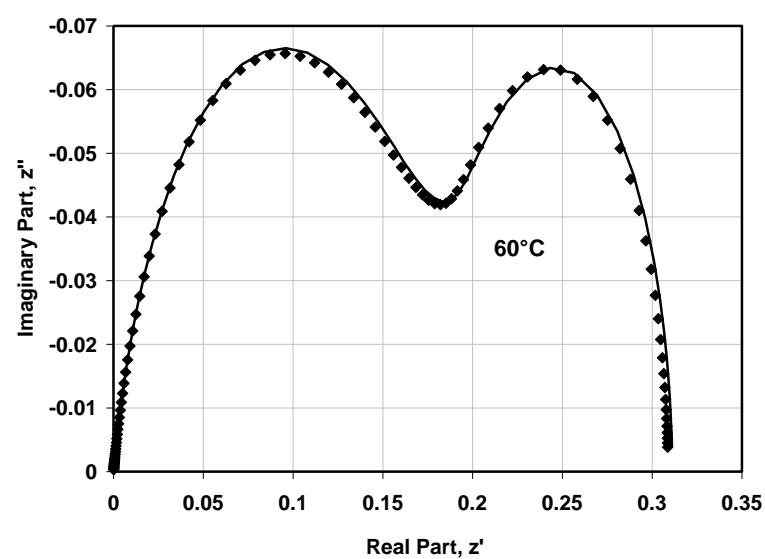

Figure 2 Comparison impedance between simulated (-) and measured ( $\bullet$ data

The results exhibit good agreement between the simulated and the measured spectra. The first arc of the impedance spectra reflects the capacitive effect due to the double layer interface between dissimilar materials and the kinetics of the ORR in the CCL. The second arc reflects the oxygen diffusion process within the CCL and is presented at low frequencies of the impedance spectrum.

\section{Transport Mechanisms in Time Domain}

The current across the thickness of the CCL in the time domain is defined from Eq. 1 by changing the $\mathrm{CPE}$ for a capacitor $\mathrm{C}$ in the distribution of charge between the electrode-electrolyte interface:

$\frac{d \bar{j}}{d x}=\frac{c_{O}^{\prime}}{c_{O}^{*}}\left(\frac{1}{R_{C}}\right) \bar{\eta}+C \frac{d \bar{\eta}}{d t}$ 
Eq.4 represents the effect of Oxygen transport through the CCL in the frequency domain. The inverse Laplace transform of Eq.4 by the Heaviside's Expansion Theorem [15] results in:

$\frac{c_{o}^{\prime}(t)}{c_{o}^{*}(t)}=1+\frac{d \bar{j}}{d x} \frac{8 \delta}{\pi^{2} z F D c_{o}^{*}} \exp \left(-\frac{\pi^{2} D}{4 \delta^{2}} t\right)$

The inverse Laplace transform of the Warburg impedance $Z_{W}$ defined in Eq.6 results in a negative expression for the concentration overpotential in the time domain. This leads us to consider that the potential of the CCL with respect to the equilibrium potential and overpotential can be represented as $E=E_{O}+\bar{\eta}$, where $E_{O}$ represents the potential at equilibrium and $\bar{\eta}$ is the overpotential of the CCL. Considering this relation, the current generation across the CCL in the time domain expressed in Eq.15 can be represented with a negative sign. As mentioned before, the first term represents the rate of charge transfer due to the distribution of oxygen and the kinetics of the ORR in the CCL. Therefore, substituting Eq.16 into the first term of Eq.15, and substituting a negative overpotential in the electrolyte network due to the relation $E=E_{O}+\bar{\eta}$ defined above, the current distribution for the CCL in the time domain is given by:

$\frac{d^{2} \bar{j}}{d x^{2}}=R_{P}\left[\frac{\bar{j}}{R_{C}+R_{M}}+C \frac{d \bar{j}}{d t}\right]$

where $R_{M}$ is defined as the mass transport resistance in the time domain such that;

$R_{M}=\frac{8}{\pi^{2}} \exp \left(-\frac{\pi^{2} D}{4 \delta^{2}} t\right) R_{W}$

Eq.17 represents the current distribution in the CCL in time domain and can be applied for any zone of the polarization curve (low, mid, high current) and can be solved using mathematical software [16]. For the specific case where $R_{M}$ is considered to be negligible either due to a high diffusion coefficient or high bulk concentration, Eq.17 will represent the current distribution in the CCL with equilibrium boundary conditions in terms of oxygen concentration. These conditions can occur for low current operation and as such, Eq.17 reduces to Equation 15 provided in the authors' previous study on low current characterisation, [12].

\section{CCL in Time Domain at High Current}

The simulated frequency response from the mathematical model is compared against an experimental impedance spectrum to obtain $R_{c}, R_{w}$, and $T_{w}$. Once validated in the frequency domain, the model is translated into the time domain by an inverse Laplace method [15]. The final stage of the study thereby 
reveals the spatio-temporal current distribution and oxygen concentration along the thickness of the CCL. To demonstrate, the data reported in the literature as shown in Table 1 and validated as shown in Figure 2 of a hydrogen-air PEFC operated at $700 \mathrm{~mA} / \mathrm{cm}^{2}$ and $60^{\circ} \mathrm{C}$ have been used for the following analysis.

\subsection{Spatio-Temporal Current and Oxygen Distribution}

After validating the impedance spectrum of the CCL, it is possible to extend the application of the theoretical model by applying Eq.17 to directly reveal both the current and oxygen concentration distributions in the CCL for a PEFC operated at a high current. Figure 3 shows the simulated current distribution of the CCL in the time domain across the CCL, where $x=0$ represents the GDL-CCL interface and $x=1$ represents the PEM-CCL interface.

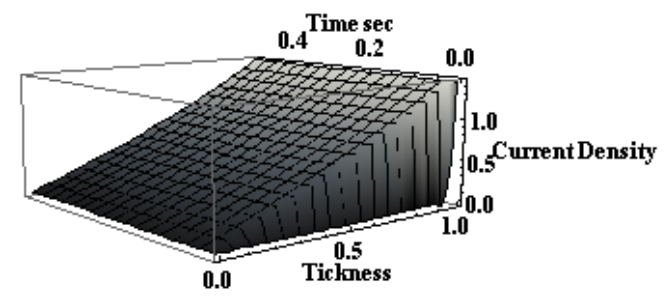

Figure 3. Simulated current distribution of the CCL in the time domain

In the authors previous study, it was demonstrated that proton resistance and capacitance can affect the transitory response of the CCL in the order of milliseconds and that only the proton resistance has an effect on the steady state low current distribution [12]. For this study, the effect of oxygen transport on the spatio-temporal current distribution of the CCL is considered. The effective diffusion coefficient $\left(1.11 \times 10^{-4} \mathrm{~cm}^{2} / \mathrm{s}\right)$ and the bulk concentration $4.2975 \times 10^{-6} \mathrm{~mol} / \mathrm{cm}^{3}$ of the oxygen were calculated from the parameters reported in Tab. 1.

Figure 4 focuses on the transitory response of the current in CCL at three locations, $x=0.1, x=0.4$, and $x=0.7$ of CCL thickness fraction (dimensionless) through the thickness of the CCL. Figure 4a shows an initial transitory response in the current distribution due to the proton resistance and the capacitance for all three locations up to $4 \mathrm{~ms}$. This response is related to the interfacial area between the electrode and electrolyte networks and has been discussed in authors' previous study [12]. Figure $4 \mathrm{~b}$ shows the response up to $0.25 \mathrm{~s}$; the results show a decrease in current in the order of $10^{-1}$ seconds due to the nonstationary oxygen diffusion process in the CCL, and that the transitory effect is most noticeable closest to the PEM boundary. 


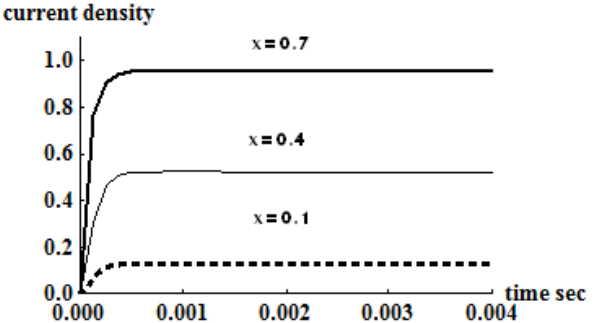

(a)

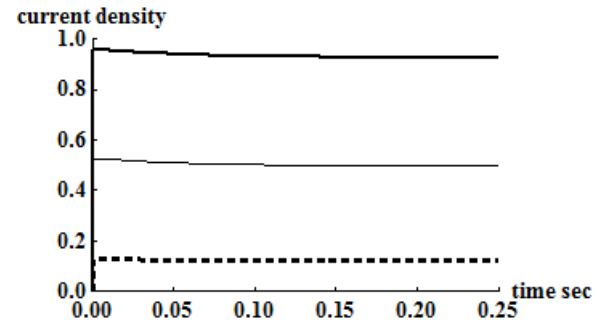

(b)

Figure 4. Simulated current distribution through the CCL

Figure 5 shows the concentration distribution through the CCL in a similar manner to the preceding sub-section. Figure 5a shows an initial depletion of oxygen concentration through the CCL, most severely closest to the PEM. This occurs due to the rapid reduction of oxygen as current is quickly established in the CCL as shown in Fig. 4a and process takes place in the order of 10ms. Thereafter, the oxygen concentration recovers to the bulk concentration value throughout the CCL at steady-state, which occurs in the order of $1 \mathrm{~s}$, as shown in Fig. 5b. Overall, therefore, Figs 4 and 5 demonstrate that the increase in current density for the three cases is proportional to the decrease in oxygen concentration in the CCL.

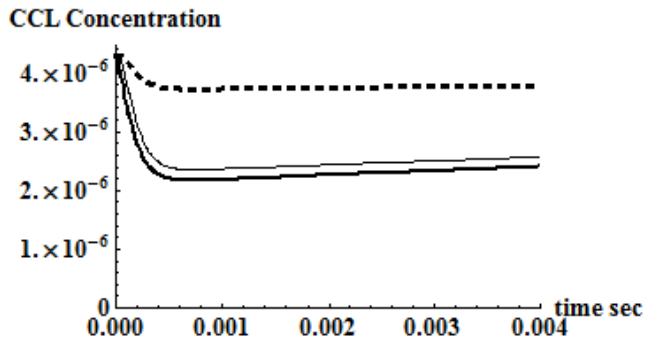

(a)

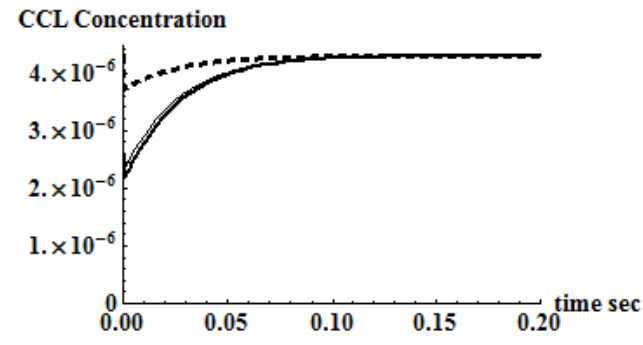

(b)

Figure 5. Simulated concentration distribution through the CCL

\subsection{Effect of Effective Diffusion Coefficient and Bulk Concentration}

It is possible to generate a broader understanding of the influence that the effective diffusion coefficient of oxygen in the CCL and the bulk oxygen concentration at the GDL boundary can have on the performance of the CCL. This therefore elucidates the effect of the mass transport resistance $R_{M}$ on CCL performance. The effective diffusion coefficients depend in part upon the nature of the tortuous porous pathways in the CCL while the bulk oxygen concentration can depend upon the conditions of the reactant supply to the cell and the physical properties of adjacent porous transport layers such as the GDL and MPL. This part of the analysis considers the effect of individually increasing the values of effective diffusion coefficient and bulk concentration as determined in Section 4.1 by one order of magnitude for effective diffusion coefficient and $500 \%$ for bulk concentration. These values were chosen because the effective diffusion can change significantly when changing the operating conditions (flooding, drying), contact pressure in the MEA assembly, CCL structure (porosity, tuortuosity), while 
the bulk concentration can change when increasing or reducing the operational gas pressure. Since the oxygen reduction reaction (ORR) occurs to a higher degree closest to the PEM as indicated by Fig. 4, the analysis is carried out at a thickness fraction of 0.7 .

Figure 6 focuses on the effect of diffusion coefficient on concentration distributions in the CCL. Figure 6a shows the initial fall in concentration to support the current drawn, while Fig. 6b shows the time to steady-state. A higher diffusion coefficient reduces the time required for the oxygen concentration within the CCL to recover such that it is in equilibrium with the bulk oxygen concentration at the GDL boundary.

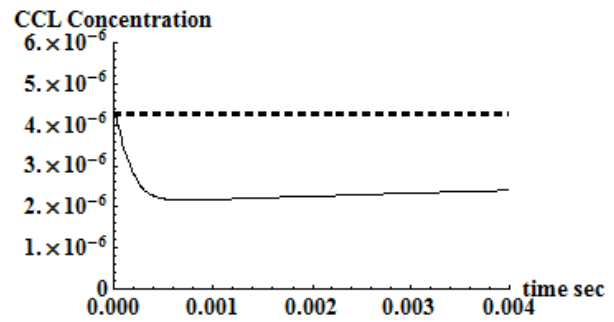

(a)

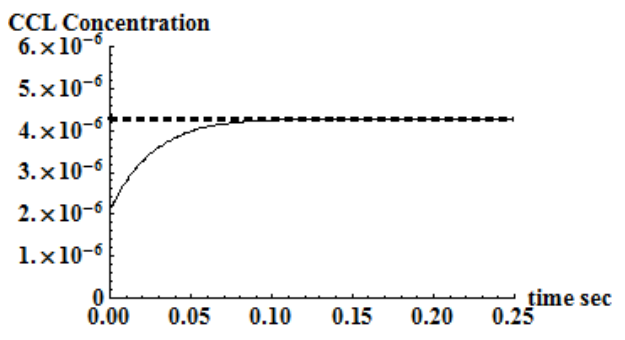

(b)

Figure 6. Effect of Diffusion Coefficient on the Transient Oxygen Concentration Distribution in the CCL, Dashed increasing by one order of magnitude, thin represents the original value.

Figure 7 shows the effect of diffusion coefficient on the transitory current density distribution in the CCL. Figure 7 a shows while the diffusion coefficient does not have a significant effect on the initial response, which is governed by $C$ and $R_{P}$, it does affect the maximum current achieved and as shown in Fig. $7 \mathrm{~b}$ the time to steady-state. By increasing the diffusion coefficient, the maximum current decreases because the oxygen consumed at CCL surface to support the current drawn is quickly replenished by the high diffusion and the time to stead-state decreases as the oxygen concentration quickly recovers to its bulk concentration value at GDL interface. The maximum current achieved represented in Fig. 7a reflects the rate at which oxygen can be brought to the electrode surface for a given set of mass-transfer conditions. A higher diffusion coefficient reduces $R_{M}$ and approaches the current response at the equilibrium conditions.

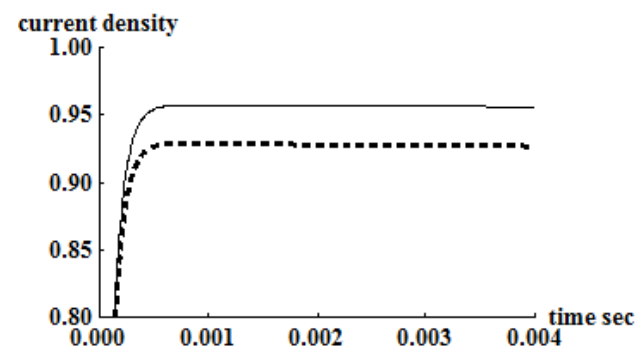

(a)

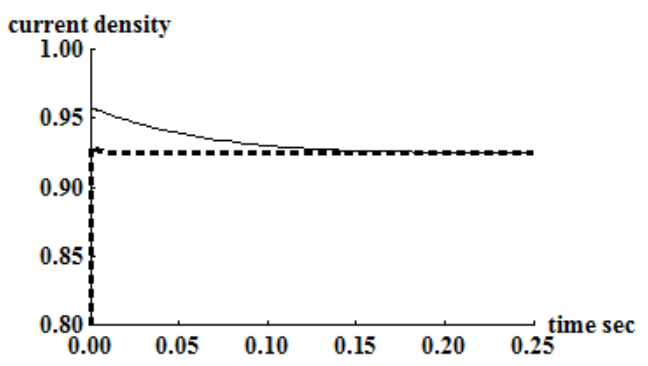

(b) 
Figure 7. Effect of Diffusion Coefficient on the Transient Current Density Distribution in the CCL, , Dashed increasing by one order of magnitude, thin represents the original value.

The simulations in Figs $8 \mathrm{a}$ and $8 \mathrm{~b}$ show the time required for the oxygen concentration in the CCL to recover to the equilibrium within the bulk concentration at the GDL interface. The recovery occurs over the same magnitude order in time for the two cases, and faster when increasing the bulk concentration.

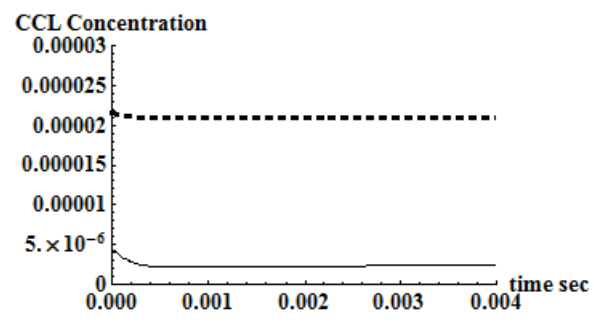

(a)

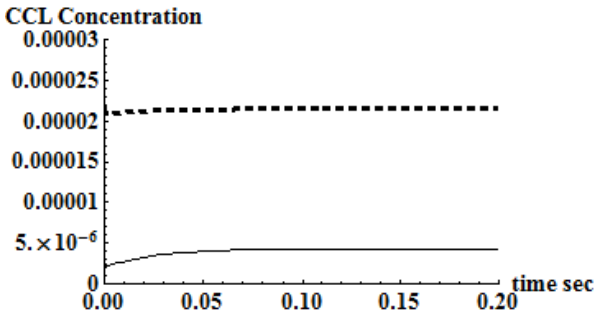

(b)

Figure 8. Effect of Bulk Oxygen Concentration on the Transient Concentration Distribution in the CCL; (a) initial response; (b) response to steady-state, Dashed increasing $500 \%$, Thin represents the original value.

Figure 9 considers the effect of bulk concentration on CCL performance; Fig. 9a demonstrates as expected that increasing bulk concentration there is a decrease in the maximum current and the transitory response of the current but over the same magnitude order in time. The same applies to the response to steady-state shown in Fig 9b compared to Fig 7b, indicating therefore that the change in current to steady-state is more sensitive to a change of oxygen diffusion than bulk oxygen concentration.

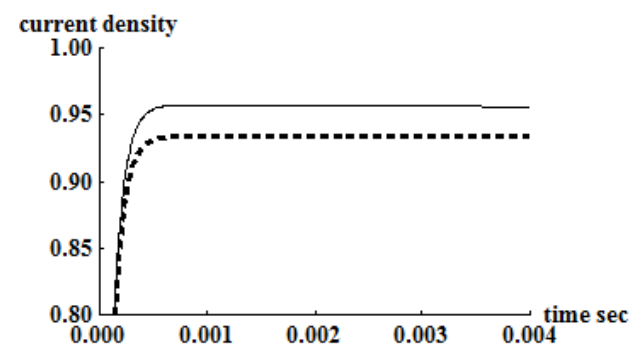

(a)

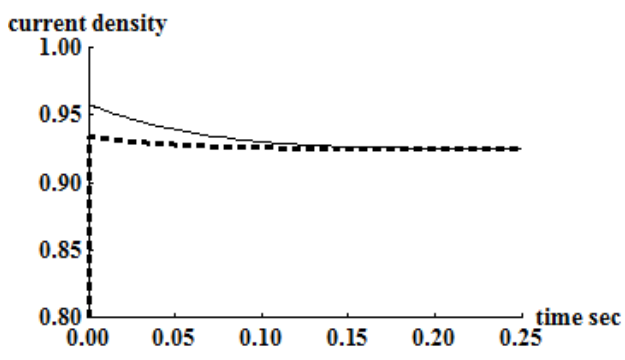

(b)

Figure 9. Effect of Bulk Oxygen Concentration on the Transient Current Density Distribution in the CCL; (a) initial response; (b) response to steady-state, Dashed increasing $500 \%$, Thin represents the original value.

\subsection{Discussion}


The effective diffusion coefficient is related to the structure of the catalyst layer, which typically features a porosity of approximately $5-15 \%$ and pore diameters of roughly $1 \mu \mathrm{m}$ [1]. The void space in the CCL can be reduced during operation due to the accumulation of product water or through design by increasing the amount of proton-conducting electrolyte impregnated within the CCL. Under these conditions, the effective diffusion coefficient can reduce correspondingly. This reduction affects the ability of oxygen to permeate into the CCL to the active sites and to recover to the bulk concentration. By maintaining the void space through design and operation, the corresponding increasing in effective diffusion coefficient can lead to diminished mass transport resistance. The bulk concentration of oxygen is largely dependant upon the operating conditions of the cell, particularly the reactant supply conditions. With a high bulk oxygen concentration, the availability of oxygen for the oxygen reduction reaction inherently increases.

The simulations show that the concentration distribution of oxygen through the CCL occurs in a nonsteady manner and recovers to the bulk concentration supplied in the GDL. The current distribution in the CCL achieves an initial maximum current as a function of the proton resistance, charge transfer resistance and mass transport resistance, as shown in Figure 4 under potentiostatic conditions. After steady-state is established in the order of one second, the magnitude of the steady-state current depends only on the proton resistance and charge transfer resistance. By increasing the effective diffusion coefficient and the bulk concentration the initial maximum current and the time to steady state decrease as shown in Figures 7 and 9. Overall, therefore, the time to reach equilibrium with respect to oxygen concentration is minimised when the PEFC is operated at low currents, or by increasing the effective diffusion coefficient or the bulk concentration.

The results provide an insight about the effect of the effective diffusion and bulk concentration on the spatiotemporal distribution of the current for the CCL. The mathematical treatment developed in this study can be applied for the different zones of the polarization curve and has demonstrated the capability of solve the spatiotemporal current distribution of the CCL based on the fundamental electrode theory and the measured frequency response of the PEFC.

\section{Conclusions}

The current study begins by solving the Fick's second law to model the rate of oxygen transport through the CCL in the frequency domain. The resulting diffusion equation is related to the current generation equation in frequency domain as defined in the authors previous study [12]. The proton resistance in the electrolyte network is subsequently considered in the expression of the current. The physical processes in the CCL such as charge-transfer resistance, double-layer capacitance, proton resistance in the electrolyte network, effective diffusion of oxygen through the CCL, and bulk concentration at the GDL boundary are taken from published experimental data [8]. 
The study began by solving the non-stationary diffusion equation expressed by Fick's second law to define the rate of oxygen distribution $c_{o}^{\prime} / c_{o}^{*}$ through the thickness of the CCL. By coupling the rate of oxygen and proton conductivity with the current distribution equation reported in the authors previous study for low current operation of the CCL in the frequency domain it was possible to establish the current distribution taking into account spatio-temporal oxygen distribution $Z_{W}$, electrode kinetics $R_{C}$, charge distribution $Y(i \omega)^{P}$ and proton resistance $R_{P}$ in the CCL.

The mathematical treatment is validated against an impedance spectrum reported in the literature for a PEFC operated at $700 \mathrm{~mA} / \mathrm{cm}^{2}$ and $60{ }^{\circ} \mathrm{C}$. The electrochemical and difussive parameters were estimated using a Randless electrical circuit. The experimental frequency response of the CCL is obtained by fitting the entire measured frequency response of the PEFC to the Randless equivalent electrical circuit. By extracting key parametric components it has been possible to represent the CCL in isolation. The mathematical model agreed well with the experimental frequency response of the CCL and was solved in the time domain by the Inverse Laplace method to establish the oxygen transport mechanisms in the time domain $R_{M}$ and to reveal the spatial and temporal distribution of the current in the CCL.

The simulations in the time domain showed that there is an initial dynamic response in the order of milliseconds due to the interfacial area between the electrode-electrolyte until a maximum current is achieved which is a function of the proton resistance, charge transfer resistance and mass transport resistance. This maximum current decreases until steady-state is established in the order of one second. The steady-state current is a function of the proton resistance and charge transfer resistance but not the mass transport resistance. The concentration distribution of oxygen was also simulated and the results revealed that the oxygen concentration consumed to support the current demanded recovered to equilibrate with the bulk concentration GDL boundary in the order of 1 second.

A further investigation on the effect of the effective diffusion coefficient and bulk concentration was considered by increasing the effective diffusion and bulk concentration by one magnitude order and $500 \%$ respectively. These increases resulted in the reduction of the initial maximum current and the time to steady state and were more noticeable for the effective diffusion coefficient. These results suggest that by increasing diffusion coefficient, it is possible to establish an equilibrium between the oxygen concentration in the CCL and the bulk of oxygen concentration supplied from the GDL and therefore to reduce the effect of the mass transport resistance in the current distribution of the CCL.

This study reveals that it is possible to interpret the frequency response of the PEFC in relation to the spatio-temporal mechanisms with the CCL. The focus of the future work is to apply this study for the diagnostic and evaluation of CCLs of different structures and composition. 


\section{Acknowledgments}

The authors thank the Mexican National Council for Science and Technology (CONACYT) for the sponsorship of the Ph.D research study of S. Cruz-Manzo (grant no. 183195)

\section{List of Symbols}

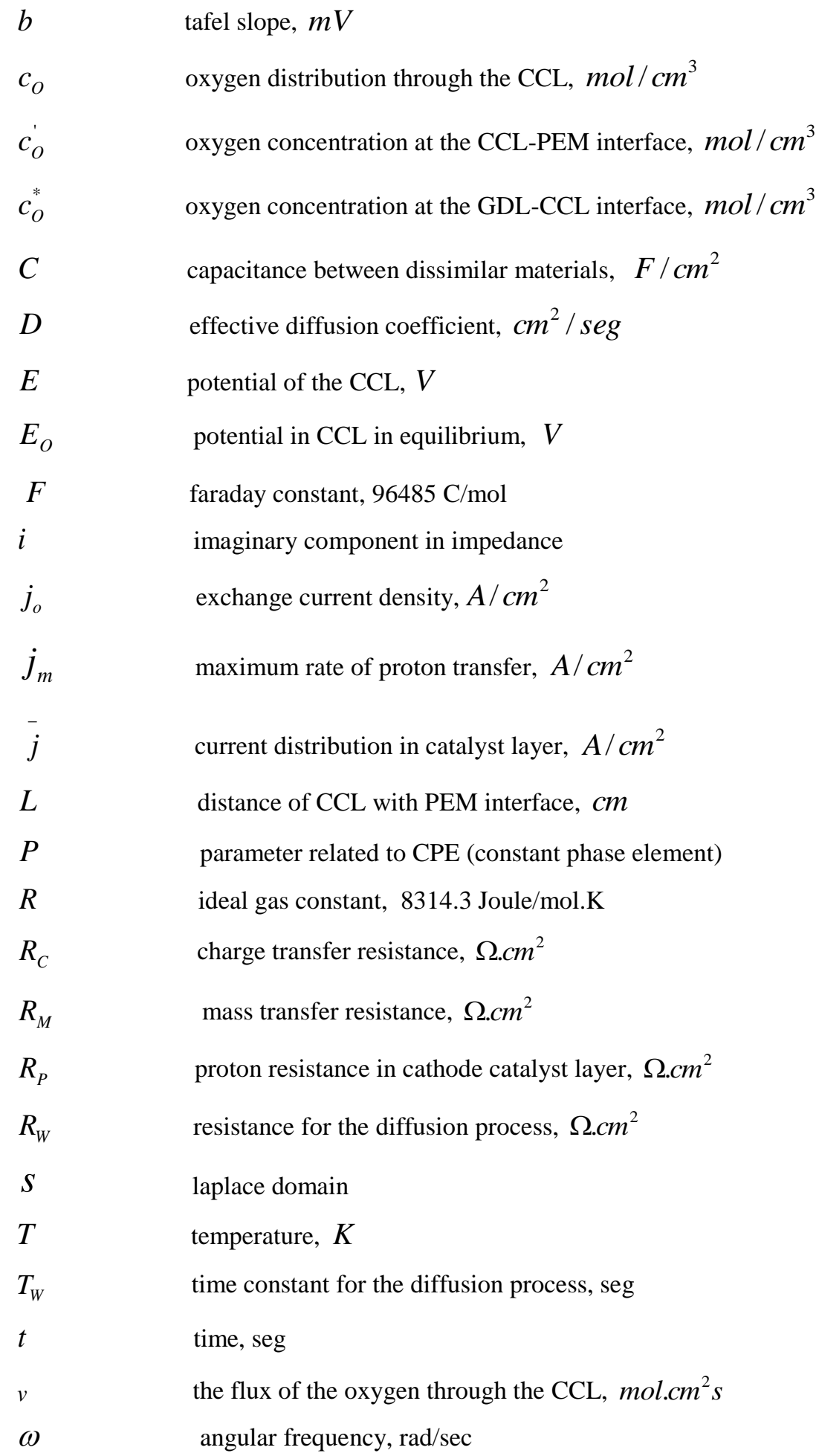




\title{
Greek
}

\author{
$\delta \quad$ finite thickness of the CCL in the through-plane direction, $\mathrm{cm}$ \\ $\eta \quad$ relationship between potential far from equilibrium and \\ equilibrium potential, $V$ \\ $\bar{\eta} \quad$ potential distribution in catalyst layer, $V$ \\ $\eta_{S} \quad$ value of voltage in activation lose where the \\ impedance is performed, $V$
}

\section{References}

\footnotetext{
${ }^{1}$ B.Sundén and M. Faghri, Transport phenomena in fuel cells,2005

${ }^{2}$ Berger, C., Handbook of Fuel Cell Technology, Prentice-Hall, Inc. / Englewood Cliffs, N.J., New York, 1968

${ }^{3}$ Amphlett, J., Baumert, R., Mann, R., Peppley, B. and Roberge, P., J. Electrochem. Soc., 142, 1, (1995)1-8.

${ }^{4}$ Larminie, J.E., Dicks, A., Fuel cell Systems Explained, John Wiley \& Sons, LTD, Chichester, England, 2000.

${ }^{5}$ Balkin, A. R., Modelling a 500 Watt Polymer Electrolyte Membrane Fuel Cells, Thesis, Faculty of Engineering, University of Technology, Sidney, Australia, 2002.

${ }^{6}$ Correa J., Farret F., Canha L, "An analysis of the Dynamic Performance of Proton Exchange Membrane Fuel Cells Using an

Electrochemical Model" 27 $7^{\text {th }}$ Annual Conference of the IEEE Industrial Electronics Society, pp 141-146, 2001.

${ }^{7}$ Kazuo Onda, Masato Nakayama, Kenichi Fukuda, Kenju Wakahara, Takuto Araki, J. Electrochem. Soc.,153,(2006),A1012A1018.

${ }^{8}$ Dzmitry Malevich, Ela Halliop, Brant A. Peppley, Jon G. Pharoah, Kunal Karan, J. Electrochem. Soc., 156, (2009), B216-B224

${ }^{9}$ S. Skale, V. Dolecek, M. Slemnik, Corrosion Science, 49,(2007), 1045-1055.

${ }^{10}$ M. Ciureanu, S. D. Mikhailenko, S. Kaliaguine, Catalysis Today, 82, (2003), 195-206.

${ }^{11}$ N. Fouquet, C. Doulet, C. Nouillant, J. Power Sources, 159, (2006), 905-913

${ }^{12}$ Samuel Cruz-Manzo, Pratap Rama, Rui Chen, J. Electrochem. Soc., 157, B400 (2010)

${ }^{13}$ Makharia R., Mathias M.F., Baker D.R., J. of the Electrochemical Soc., 152, 5, (2005), A970-A977

${ }^{14}$ U. Cano-Castillo, A. Ortiz, S. Cruz, L. G. Arriaga and G.Orozco, ECS Transactions PEMC 6, V3, Issue I, pp931-939, 2006, Editors: T. Fuller et al.

${ }^{15}$ Lokenath D., Dambaru B., Integral Transforms and Their Applications, 2007, Chapman

${ }^{16}$ Wolfram Mathematica 7, http://www.wolfram.com/products/mathematica/index.html, last accessed May 2010
} 